\title{
Falla en la equivalencia funcional: Problema conceptual más que metodológico.
}

\author{
Failures in the functional equivalence: Conceptual issue rather than methodologic one
}

\author{
Gelacio Guzmán-Díaz ${ }^{a}$, Jesús Cisneros-Herrera ${ }^{b}$
}

\begin{abstract}
:
This work aims at showing some conceptual failures in the study of functional equivalence (Tonneau, 2004), also called transfer of function in the approach of Relational Frame Theory (RFT) (Hayes, Barnes-Holmes, \& Roche, 2001). This analysis is based on the work "Sitting On the Word Chair: Behavioral Support, Contextual Cues and the Literal Use of Symbols", by Francois Tonneau, Nadjelly Kim Abreu and Felipe Cabrera (2004). Althoug such work does not encompass, nor exhaust the study of functional equivalence, it is assumed that it can help to illustrate how empirical findings regarding this issue come from a conceptual failure rather than from a methodological one.
\end{abstract}

\section{Keywords:}

Functional equivalence, Transfer of Function, Relational Frame Theory, Verbal Behavior, Language

\section{Resumen:}

El presente trabajo intenta mostrar algunas deficiencias conceptuales dentro del estudio de la equivalencia funcional (Tonneau, 2004), o también llamada, por la perspectiva de la Teoría de los Marcos Relacionales (RFT), transferencia de función (Hayes, BarnesHolmes, \& Roche, 2001). Para este análisis se tomó como punto de partida el trabajo "Sentarse sobre la palabra silla: apoyo conductual, señales contextuales y el uso literal de símbolos", realizado por Francois Tonneau, Nadjelly Kim Abreu, y Felipe Cabrera (2004). Si bien dicho trabajo no engloba, ni agota el estudio de la equivalencia funcional, se cree puede servir para ejemplificar como los hallazgos empíricos con respecto a este tópico, son derivados de una insuficiencia conceptual más que metodoló gica.

\section{Palabras Clave:}

Equvalencia funcional, trasferencia de función, Teoría de Marcos Relacionales, Conducta verbal, Lenguaje

\section{Introcucción}

El presente trabajo intenta mostrar algunas deficiencias conceptuales dentro del estudio de la equivalencia funcional (Tonneau, 2004)(1), o también llamada, por la perspectiva de la Teoría de los Marcos Relacionales (RFT), transferencia de función (Hayes, Barnes-Holmes, \& Roche, 2001)(2). Para este análisis se tomó como punto de partida el trabajo "Sentarse sobre la palabra silla: apoyo conductual, señales contextuales y el uso literal de símbolos", realizado por Francois Tonneau, Nadjelly Kim Abreu, y Felipe Cabrera (2004)(3). Si bien dicho trabajo no engloba, ni agota el estudio de la equivalencia funcional, se cree puede servir para ejemplificar como los hallazgos empíricos con respecto a este tópico, son derivados de una insuficiencia conceptual más que metodológica.
El lenguaje como una segunda naturaleza y su relación con la psicología.

El lenguaje humano junto con la cognición, son dos temas que recientemente han ocupado gran interés dentro de la psicología de la conducta. Tanto investigadores teóricos como empíricos han enfocado sus esfuerzos en conocer aspectos del lenguaje como su adquisición y su uso.

$\mathrm{Si}$ bien el lenguaje no es un término técnico de la psicología, esta lo ha adoptado y lo ha abordado desde una postura conductual. En el caso particular del análisis experimental de la conducta, el estudio científico del lenguaje fue posibilitado por la introducción por parte de Skinner (1957)(4) del concepto de conducta verbal, es decir, aquella conducta que es reforzada a través de la

\footnotetext{
autor de Correspondencia, Universidad Autónoma del Estado de Hidalgo, Escuela Superior de Atotonilco de Tula, ORCID: https://orcid.org/0000-0003-4869-8658,Email: gelacio_guzman@uaeh.edu.mx 
mediación de otras personas: un escucha entrenado por la "comunidad verbal" para reforzar la conducta del hablante.

La aproximación skinnereana al análisis experimental del lenguaje, entendido como conducta verbal, abrió la puerta para que la psicología conductual diera cuenta de temas como el propio lenguaje y el pensamiento. Dicha aproximación ha recibido varias críticas, entre las que destacan, la extrapolación de los métodos empleados del estudio del comportamiento animal al comportamiento humano, la insuficiencia para dar cuenta episodios lingüísticos complejos y por la ineficaz aproximación al estudio funcional de lenguaje (véase Ribes 1990a(5), Hayes, Barnes-Holmes, \& Roche, 2001)(2).

Desde la aproximación operante, se adoptó la postura de concebir al lenguaje como un entramado de estímulos verbales. De esta forma, las palabras (estímulos verbales) daban pie para la ocurrencia de respuestas operantes. Sin embargo, todavía faltaba explicar cómo estas respuestas o conducta operante podía ser controlada por estímulos que no tenían morfología lingüística (por ejemplo, detenerse frente a la luz roja de un semáforo).

A este respecto, Skinner (1969)(6) propone distinguir entre dos tipos de conducta, la conducta que es moldeada por las contingencias y la conducta gobernada por reglas. La conducta moldeada por las contingencias que se distingue de la conducta gobernada por reglas en términos de la exposición directa a las contingencias versus el control antecedente ejercido por la especificación de las contingencias tipo regla. Desde la perspectiva operante la regla es concebida como un estímulo discriminativo construido por el mismo individuo o por otros individuos. De esta forma la conducta gobernada por reglas es conducta controlada por estímulos discriminativos.

La formulación entre estos dos tipos de conducta no quedo exenta de críticas, sobre todo por el hecho de que la distinción entre ellas parce obedecer a criterios operacionales, haciendo imposible distinguirlas en términos funcionales. (Véase Ribes 1990b(7), Hayes, Barnes-Holmes, \& Roche, 2001(2)).

Sidman (1971)(8) demostró bajo su procedimiento de equivalencia de estímulos como era posible el surgimiento de relaciones entre estímulos (aun cuando tuviesen morfología diferente), y como a partir de este procedimiento podrían emerger nuevas relaciones entre estímulos no entrenados.

El trabajo de Sidman consistió, básicamente, en enseñar a un niño discapacitado a aprender a igualar palabras habladas con imágenes y palabras habladas con palabras escritas. Cada relación "no entrenada" se le llamo relación derivada de estímulos, para distinguirla de aquella relación explícitamente entrenada.

Las aportaciones de Sidman, aun cuando enraizadas en la psicología operante, resultaron ser aproximaciones consistentes, factibles y atractivas para el estudio de la adquisición y el uso del lenguaje como comportamiento.

Bajo el arropo de los hallazgos de Sidman, una visión crítica a la teoría del lenguaje de Skinner, y la incorporación de la psicología interconductual de Kantor (1959)(9), surge la Teoría de los Marcos Relacionales (RFT).

La RFT, tal y como sus autores la definen, se concibe como una aproximación basada en un tipo de pragmatismo llamado contextualismo funcional. Pone énfasis en el contexto, le da importancia a la historia individual y reconoce la importancia de todos los eventos involucrados en el contexto.

Uno de los conceptos fundamentales dentro de la RFT, es el de trasformación de funciones o transferencia de función. La transferencia de función se da cuando, las funciones de un estímulo son transferidas a otro como respuesta relacional ejercida sobre la relación por el contexto.

Si bien, la RFT surge como una alternativa para el estudio del lenguaje que intenta superar viejas aproximaciones como la de Conducta Verbal de Skinner. Varios detractores, opinan que dicha teoría, sigue siendo operante y cuyos fenómenos explicativos pueden ser entendidos bajo la perspectiva del condicionamiento.

Uno de los críticos a la posición operante de la RFT, ha sido, Francois Tonneau. Dicho autor se ha interesado, entre otras cosas, por como las palabras adquieren su significado, es decir como una palabra está ligada con su referente. Para Tonneau (2004) una propiedad importante de la comprensión verbal es que los estímulos verbales tienden a ser funcionalmente equivalentes a sus referentes, dos eventos A y B pueden ser funcionalmente equivalentes si estos tienen los mismos efectos sobre la conducta.

Para Tonneau (2004)(1) una posible respuesta a esta equivalencia funcional de estímulos verbales y sus referentes involucra un proceso parecido al condicionamiento pavloviano. Todos los puntos de vista pavlovianos, dice el autor, acerca de la respuesta simbólica ponen énfasis en el fenómeno de la equivalencia funcional y la dependencia sobre correlación de estímulos. Sin embargo, Tonneau (2004) (1) y Tonneau y Cols. (2004)(3) aclaran que a veces las palabras y sus referentes no verbales llegan a fallar en ser funcionalmente equivalentes. Desde el punto de vista pavloviano, diferentes factores pueden explicar estos desaciertos. Por ejemplo el contexto en el que están los estímulos.

Como podemos dar cuenta, tanto Tonneau como los autores de la RFT enfatizan la importancia de la equivalencia funcional como fenómeno que podría dar cuenta de cómo las palabras funcionan como sustitutos de un mundo físico o como una segunda naturaleza, es decir 
como las palabras adquieren su significado, aunque sus posiciones sean distintas.

\section{Falla en la equivalencia funcional deficiencias metodológicas derivadas de deficiencias conceptuales.}

Como se mencionó anteriormente, en ocasiones se ha encontrado, en el trabajo empírico de la equivalencia funcional, ciertos resultados desfavorables hacia esta postura propuesta por Tonneau. En general, se ha argumentado una falta de equivalencia funcional entre estímulos verbales y no verbales usando modelos pavlovianos de comprensión verbal. El argumento es que, bajo estos procedimientos, son múltiples las ocasiones en las que no nos comportamos hacia una palabra escrita o hablada de la misma forma que nos comportamos hacia un objeto u evento.

Resaltando la importancia del contexto en este sentido, Tonneau y cols. (2004)(3) evaluaron si la equivalencia funcional entre la palabra y el objeto dependía del apoyo conductual y de los estímulos contextuales. Hay que hacer notar que la RFT, igualmente habla de la importancia del contexto en que ocurren los eventos de estímulos como factor necesario para que se dé la equivalencia funcional. En el estudio de Tonneau y cols. (2004)(3), se utilizó la palabra escrita "silla" para que funcionara como estímulo equivalente a una silla de verdad, es decir, que los sujetos experimentales se sentaran sobre la palabra. Para ello, se hizo uso del contexto, que para el caso de este estudio, consistió en la escenografía que acompañaba a la palabra silla. Para el caso donde el contexto favorecía la equivalencia funcional la palabra "silla" iba acompañada de una escenografía que representaba pictóricamente el interior de una casa y para la condición desfavorable la escenografía consistió en garabatos que no representaban nada. En ambas condiciones se utilizaron niños como sujetos experimentales y la situación experimental consistió en ponerlos en el área de investigación sin ningún tipo de instrucción, se esperaba que el participante actuara libremente sin instigaciones a comportarse de cierta forma 0 a realizar ciertas actividades con el material presente.

En general, los autores encontraron evidencia favorable al uso del contexto aunque no contundente. Los autores se excusan aclarando que, mostrar que el grado de equivalencia funcional entre un símbolo y su referente puede incrementar gracias al contexto, esto no demuestra concluyentemente que la conducta simbólica dependa de procedimientos pavlovianos. Afirmar esto, dicen los autores, sería prematuro ya que es más complejo de lo que usualmente se asume.

Dentro de las deficiencias metodológicas se pueden marcar varias, no obstante, muchas de ellas se deben a una falta de control experimental o por la situación experimental empleada. El problema subyacente de estas, sin embargo, se deben al marco conceptual que cubre a la equivalencia de función.

En primer lugar, podríamos pensar en, porque igualar una palabra con un objeto. La palabra escrita tiene como referente a la palabra vocalizada, la palabra escrita es una forma de representar una vocalización. Por lo que la palabra escrita, en este caso "silla", no tiene como referente a un objeto real, es decir una silla real. De este modo, si cada palabra tuviese un objeto referente, hablaríamos de una correspondencia uno a uno, habría tantas cosas como similar número de palabras.

Entonces, ¿Cómo las palabras adquieren su significado?, tanto Tonneau como los autores de la RFT, tienen razón en brindar al contexto gran parte de la responsabilidad en la funcionalidad que adquieren los eventos de estímulo, incluyendo claro las palabras. Sin embargo, el contexto no se refiere solo a los eventos físicos que acompañan o contextual al evento estimulo objetivo.

La funcionalidad de la palabra hace referencia al concepto. La función de la palabra es condicional a su ámbito y criterio de uso, es decir, no puede desvincularse de la situación ni del contexto lingüístico en que la palabra ocurre como parte o elemento de una frase o expresión. Las palabras solamente tienen una función en el contexto de una expresión o de un acto (Ribes, 2006)(10).

El significado o sentido de las palabras, son las actividades en las que están incorporadas. Por ello es que hablar del significado de las palabras es referirse a su uso en contexto. Es decir, a sus funciones prácticas en situación (Ribes, 2011)(11).

Es por ello, que la palabra "silla" o cualquier otro evento de estímulo que se quisiera funcionara como una silla real, más que estar rodeada por elementos contextualizadores, tendría que tener la función del objeto que se pretende igualar. Si no me siento sobre la palabra silla es simplemente porque esta no satisface las condiciones de uso que tiene una silla real.

De acuerdo con Ribes (2006)(10), las palabras y los actos en si carecen de sentido o función. Es su ocurrencia como actos y expresiones apropiadas y pertinentes a una situación lo que les da sentido. El grado en el que dichos actos y expresiones pueden ser funcionales en distintas situaciones depende del juego de lenguaje, por así decirlo, es decir, no depende de los objetos y personas per se con los que se interactúa, sino del criterio que lo regula o determina dicha interacción.

Por otro lado, cuando se dice que entender, tener o utilizar apropiadamente un concepto, se está hablando de que hemos logrado ajustarnos a una situación en términos de los límites funcionales de pertinencia de nuestras acciones (Ribes, 2006)(10). En el caso de la investigación revisada la situación no imponía los límites funcionales 
para que los participantes se comportaran, sino que era una situación de contingencias abiertas. De esta forma podemos decir que no había criterio a lograr. A menos que el criterio fuese sentarse. Que debido a los 15 a 20 minutos que duraba la sesión experimental no sería extraño que los participantes se sentaran más por fatiga que por la funcionalidad del objeto.

\section{Conclusiones}

Desde la perspectiva de Ribes (2006) (10), los conceptos hacen referencia a como, mediante comportamiento, un individuo reconoce, actúa con base en, aplica y formula criterios de ajuste respecto de atributos y propiedades variantes en una misma situación o entre situaciones. Las transiciones de función de un mismo conjunto de atributos o propiedades en los objetos y símbolos, se aprenden en relación directa mediante el ejemplo, las consecuencias y la practica social del lenguaje, y parte de ese aprendizaje consiste en distinguir entre los indicios que componen el criterio de pertinencia de aquellos que solo lo acompañan en cada situación.

En conclusión, no hay una relación univoca entre objeto y palabra, una palabra puede hacer referencia a múltiples objetos dependiendo del ámbito en el que tenga lugar. Así, por ejemplo, la palabra gato puede hacer referencia a un animal si es que estoy en una tienda de mascotas o a una herramienta si estoy en un taller mecánico. Así también, un objeto puede funcionar tener múltiples funciones dependiendo del contexto en que se encuentre, por ejemplo, una silla funciona como tal si necesito sentarme a leer, pero esa misma silla puede ser una escalera si necesito alcanzar un objeto fuera de mi alcance.

El que se dé o no una equivalencia de función, si bien depende de la metodología empleada para dar cuenta de ello, esta debe ir arropada por una consistencia conceptual y comprensiva acerca del fenómeno de la significación.
6. Skinner BF. Contingencies of reinforcement: A Theoretical analysis. Nueva York: Appleton Century Crofts; 1969.

7. Ribes E. Problemas conceptuales en el análisis del comportamiento humano. México: Trillas; 1990.

8. Sidman M. Reading and Auditory-Visual Equivalences. J Speech Hear Res. marzo de 1971;14(1):5-13.

9. Kantor JR. Interbehavioral psychology: A sample of system construction. Granville: Principia Press.; 1959.

10. Ribes E. Categorías, conceptos y conducta: reflexiones teóricas. Rev Lat Pensam Leng. 2006;15(1):5-23.

11. Ribes E. La noción de juego de lenguaje como instrumento heurístico de la Psicología. Doc No Publ. 2011;

\section{Referencias}

1. Tonneau F. Verbal understanding and Pavlovian processes. Behav Anal Today. 2004;5(2):158-69.

2. Hayes SC, Barnes-Holmes D, Roche B. Relational frame theory: A postskinnerian account of human language and cognition. Nueva York: Kluwer Academic/Plenum Publishers.; 2001.

3. Tonneau F, Abreu, K., Cabrera, F. Sitting on the word "chair", Behavioral support, contextual cues, and the literal use of symbols. Learn Motiv. 2004; 35:262-273.

4. Skinner BF. Verbal behavior. Acton, Massachussets: Copley Publishing Group; 1957.

5. Ribes E. Psicología general. México: Trillas; 1990. 